Monatsschrift f. Geburtshülfe u. Gynäkologie 1917;46:485-489

\title{
Paul Krömer †
}

Von A. Martin

Tief erschüttert stehen wir vor der Katastrophe, welche unseren jungen Kollegen in Greifswald mitten aus einer glänzenden Tätigkeit hinweggerafft hat. Den treuen Worten seines Schülers Schroder, der während der Krankheit als einziger die khnische Arbeit zu leisten hatte, drängt es mich, einige Worte freundhcher Anerkennung für den Ver-storbenen hinzuzufügen. Als ich Krömer zuerst in der Pfannenstielschen Klinik in Gießen kennen lernte, fesselte mich sein liebenswürdigesWesen und der Ernst, mit dem er sich seinen Äufgaben hingab. Mit wachsendem Interesse habe ich seine tiefgründigen, überaus fleißigen Arbeiten ver-folgt und konnte mit Freund Pfannenstiel lebhaft sympathisieren, wenn er mir von dem festen Vertrauen auf die für unser Fach zu erwartenden großen Leistungen Kramers sprach. Nur zu früh wurde ihm insbesondere wie uns alien Pfannenstiel entrissen. Es War ein besonderer Glücks-zufaiï, daß er $1 / 8$ die Beiliner Klinik kam und hier unter der Leitung Bumms noch das große Material der Berliner Klinik zu bearbeiten Ge-legenheit fand. Mit welchem Erfolg er nach dieser Ausbildung das akademische Lehramt in Greifswald übernahm, da von sprechen nicht 3-*

486

Pavl Krömer $\dagger$. nur die von Schroder gerühmten Erfolge. Ich weiß, daß Krömer mit einer Fülle von Arbeiten beschäftigt war, in denen die Ernte aus diesen Arbeitsjahren eingebiacht werden sollte. Schon der Kñeg brachte die Hemmung, dann seine Krankheit, und nun läßt sein fiüher Tod eine FüUe von Gedanken und Arbeit, mit denen Krömer sich trug, mit $1 / 8 \mathrm{~m}$ ins Grab sinken. Es genügt hier auf die nachstehende Bibliographie hinzuweisen, urn die Vielseitigkeit von Kramers Arbeit zu beweisen.

Wir trauern um ihn mit seiner hochverehrten Gattin, die ihm eine überaus treu fürsorgende Weggenossin war, mit seinen Schülern und eine.i. überraschend großen Kreise dankbarer Klienten. Krömer hat seinen Namen in die Geschichte unførts Faches in lapidaren Zügen eingetragen. Seine Arbeit wird seinen Namen allezeit lebendig erhalten.

Have! Have! A. Martin.

[Biilio graphic. |1

1895: 1. Beitrag zur Kastrationsfrag0. Zt, sc $1 / 8$. f. Psychol. - 1898: 2. Anaton,ie der Bern.oide und Teraton.e des Eierstocks. In Pfannenstiels Erkrankuní: en des Eierstocks (Veits Handb. d. Gyn.) - 3. Über die Histo-genese der Bern.oidkyston. $\theta$ und Teraton.e des Eierstocks. Arch. f. Gyn. Bd. 57. (Inaug.-Diss.)--1900: 4. Zur KenntnisdesLithopädion. Munch, n.ed. Woch. No. 42. - 1901: 5. Ein seltener Mischtun,or des Ovariun.s. Aug. rr,ed. Zbl. - 1902: 6. Klinische und anato $\pi$, ische Untersuchungen über den Gebärn.utterkrebs. Arch. f. Gyn. Bd. 65. -1903: 7. Die ïechnikder Äther-narkose an der Frauenklinik zu Gießen. Zbl. f. Gyn. - · 8. Über die Lyn ,phorgane der weiblichen Genitalien. Men. f. Geb. u. Gyn. Bd. 18. - 9. Wann soil man Geschwü1/1/8te des Uterus und des Eierstocks vaginal entfernen und in welchen Fallen ventral ? Mon. f. Geb. u. Gyn. Bd. 18. - · 10. Über Extra-uteringravidit.ät. Btsch. rr,ed. Woch. No. 9. - 11. 
Untersuchungen über die tub3/8re Eieinbettung. Arch. f. Gyn. Bd. 68. - 12. Berr,onstration des Wachsn, odells èines jungen $\mathrm{n}$, enschlichen En, bryo. Verhandl. d. Btsch. Ges. f. Gyn. Würzburg. - 1904: 13. Die Lyn,phorgane der Weiblichen Genitalien und ihre Veränderung bei rr,alignen Erkrankungen des Uterus. Arch. f. Gyn. Bd. 73. (Habilitationsschrift.)--14. Pflege der frühgeborenen Kinder. Btsch. rr,ed. Woch. No. 7. - 15. Über sog,enannte Melaena neona-torum. Mon. f. Geb. u. Gyn. Bd. 20. - 16. Bie Prophylaxe der Wochenbett-rr.orbidität in der Schwangerschaft. Mon. f. Geb. u. Gyn. Bd. 20. --27. Scho-nende oder forcierte Entbindung bei Eklan,psie. Zbl. f. Gyn. No. 19. - - 18. Bie Prophylaxe der Wochenbettsrr.orbidität an der Gießener Entbindung sanst.alt. Zbl. f. Gyn. No. 45. - 19. Erfolge und Aussichten der künstlichen Frühgeburt. Mon. f. Gob. u. Gyn. Bd. 20. - 20. Über Chorion-epitheliorr.a rr, alignum,. Btsch. rr,ed. Woch. No. 52. - 21. Barmverschluß. Mon. f. Geb. u. Gyn. Bd. 20. - 1905: 22. Bericht über den wiederholten GeburtsVerlauf bei einer Patientin n, it schrägverengtem, Naegeleschem Becken. Mon. f. Geb. u. Gira. Bd. 21. - 23. Über rr, aligne Tuboovarial-tun,oren. Mon. f. Geb. u. Gyn. Bd. 22. - 24. Über den Bau der 1r,ensch-lichen Tube. Mit Ben,onstrationen von Wachsir,odellen. Zbl. f. Gyn. No. 42. -' 25. Geburtsstörungen durch narbige Scheiden- und Vulvaver-engerung. Btsch. med. Woch. No. 31. - 1906: 26. Bie Vereinfachung der Gehirnfaserungsrr, ethod $\theta$ und ihre Verwendbarkeit für den Unterricht. Anat. Hefte v. Merkel u. Bonnet. Bd. 31. H. 95. - 27. Bas Biersche Stau-ungsverfahren in der Gynäkologie. Zbl. f. Gyn. No. 4. - 28. Zur Frage der Beckenerweiterung bei derHebosteotomie. Zbl. f. Gyn. No. 8. - 29. Seltene

Paul Kröir.er $\uparrow$. $\quad 487$

Tumoren des Urogenitaltraktus. Mon. f. Geb. u. Gyn. Bd. 23. - 30. Unter-suchungen über den Bau der menschüchen Tube zur Klärung der Divertikel-frage irittelst Modellrekonstruktion nach Born. Leipzig. S. Hirzel. - 31. Beckenerweiternde Operationen. $\Gamma>$ tsch. rr.ed. Woch. No. 22. - 32. Drei Beobachtunren von spondylolysthetischem Beckon. Mon. f. Geb. u. Gyn. Bd. 24. 1907: 33. Der heutige Stand der Wochenbettpflege. Med. Woch. 1-4. - 34. Über einige seltene Forrr, en der Genitaltuberkulose des Weibes. Mon. f. Geb. u. Gyn. Bd. 26. - 35. Über Extrauteringravidität und Blasen-rr.ole. Dtsch. rr,ed. Woch. No. 22. - 36. Die VerWertung des histologischen Bildes für die Therapie des Uteruskarzinorr,s. Verhandl. d. Dtsch. Ges. f. Gyn. Bd. 22. - 37. Klinische Beobachtungen über Ätiologie und Therapie des Chorionepitheliorr.s. Dtsch. med. Woch. No. 31. - 38. Störungen der Dezidualspaltung am Reflexa-Basaliswinkel und ihre Beziehungen zu Abort, Placenta circumvallata ixnd exochorialer Fruchtentwicklung. Zbl. f. Gyn. No. 5. - 39. Blasenschutz während und nach der Hebosteotomie. Zbl. f. Gyn. No. 24. 40. Über Versuche, den prirr,ären Verlauf und die Dauer-resultate der Hebosteotonie zu bessern. Beitr. z. Geb. u. Gyn. Bd. 20. - 41. Operative HOilung eines Anus anomalus vulvovestibularis bei einem. Säugling. Munch, med. Woch. Bd. 54. No. 10. - 42. Über Schwierig-keiten bei der Diagnose von Abdominaltumoren. Mitt. a. d. Grenzceb. d, Med. u. Chir. Bd. 18. H· 3. - 43. Über die Behandlung der Neben-verletzungen bei der Hebosteotomie. Zbl. f. Gyn. Bd. 31. - 1908: 44. Er-folge und Aufgaben der modernen Geburtshülfe und Gynäkologie. Ther. Monatsh. No. 7. (Antrittsvorlesung in Berlin.) - 45. Die ovulogenen und stromatogenen Neubildungen. Aus 17/8krankunpen des Ei.ersfcockes in Veits Handb, d. Gyn. 1908. - 46. Die Erfahrungen der TJniversitäts-Frauenklinik an der Charité über die Pixbeotomie. Berl. klin. Woch. No. 22. -47. Vulvovaginitis gonorrhoica bei kleinen Mädchen. Dtsch. med. Woch. No. 39. - 48. Gelatinebehandlung von Metrorrhagien. Dtsch. med. Woch. No. 50. - 4 49. Ovarialbefund bei Uteruskarzinom. Zbl. f. Gyn. No. 44. - 50. Über den Einiluß der Wundbehandlung auf den Heilungsverlauf der abdominalen Krebsoperation. Mon. f. Geb. u. Gyn. Bd. 28. - 1909: 51. 
Puerperaler Uterus mit Weit vorgesehrittenem Hats-krebs. Zbl. f. Gyn. No. 17. -- 52. Zur abdominalen Radikaloperation bei Karzinom. Zbl. f. Gyn. No. 17. -- 53. Zur Unterscheidung von sohden und cystischen Tumoren. Zbl. f. Gyn. No. 17. - 54. Die Differentialdiagnose der Abdominaltumoren und die Grenzen ihrer Leistungsiähigkeit. Ztschr. f. Geb. u. Gyn. Bd. 64. - • 55. Über die Einschränkung der Narkose bei gynakologischen Encheiresen. Dtsch. Gyn.Kongreß zu Straßburg. - - 56. Über die Indikationen zur chirurgischen Behandlung des Puerperal-fiebers. Dtsch. Gyn. -Kongreß zu Straßburg. - 57. Metroph $1 / 8$ bitis. Verhandl. d. Dtsch. Ges. f. Gyn. Bd. 13. - 58. RezidiVoperation nach Ovarialkystom. Ztsohr. f. Geb. u. Gyn. Bd. 64. - 59. Vaginalpessar zur Befestigung von Drainröhren und Dauerkathetern. Verhandl. d. Dtsch. Ges. f. Gyn. Bd. 13.-· 60. Die Behandlung unfreiwilliger Ureterläsionen und unterbindungen. Verhandl. d. Dtsch. Ges. f. Urol. - 61. Kolpeurynterurinal. Verhandl. d. Dtsch. Ges. f. Gyn. Bd. 13. - 62. Zum Einfluß der Geburt auf den Levator-spalt. Verhandl. d. Dtsch. Ges. f. Gyn. Bd. 13. - 63. Eine einfache Vo · richtung zur Ent· wicklung d,er kalten Schlinge als Ergänzung zum Ureteren-cystoskop. Ztschr. f. gyn. Urol. Bd. 1. - 64. Nekrolog auf Pf\&nnenstiel. Arch. f. Gyn. Bd. 89. - 65. Die Pensionierung der Hebammen in den nörd488 Paul Kröm $\beta \mathrm{r} \uparrow$.

lichen Bundesstaaten. Ztschr. f. d. ges. HebammenWesem 12. - 66. Atiologie und Therapie des Lupus esthioir.Onos vulvae. Char.-Ann. 34. Jahrg. - 67. Die Verwendbarkeit des Burrischen

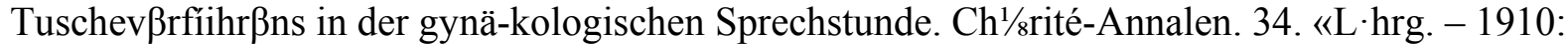
68. Blaseninsuffizienz nach Pubeotomie ohne Fistel und ihre operative Be-handlung. Ztschr. f. Geb. u. Gyn. Bd. 65. - 69. Über die Entwicklung des Begriffes und die Bodeixtung der Phlegmasia alba dolens. Arch, f. Gyn. Bd. 92. - 70. Die Berechtigung der Pubeotorr,ie. Dtsch. rr,ed.Woch. No. 36. - 71. Prolaps eines graviden Uterus in eine Ventralhernie. Dtsch. rr,ed. Woch. No. 46. - 72. Fall von Esthioméne. Ztschr. f. Geb. u. Gyn. Bd. 66. - 73. Therapie des Uteruskrebses. Dtsch. med. Woch. No. 49. -- 74. Anorr, alie der Nabelschnur; Härr, atorr.e durch Platzen eines Varix der Nabelschnur-vene. Ztschr. f. Geb. u. Gyn. Bd. 67. - 1911: 75. Über die Anwendungs-breite der Lokalanästhesie in der Gynäkologie und Geburtshülfe. Berl. klin. Woch. No. 38. - 76. Genitalbßfchwerden bei Sklerodermie. Dtsch. rr.ed. Woch. No. 6. - 77. Ein Doppelluftkissen für den Operationstisch. 83. Vers. dtsch. Naturf. u. Ärzte. -· 78. Durch Stieltorsion koir,plizierte Ovarialturnoren. Ztsc $1 / 8$. f. Geb. u. Gyn. Bd. 68. - 79. Uterinstifte. Ztschr. f. Geb. u. Gyn. Bd. 68. - - 80. Zottentuir,or der Blase. Ztschr. f. Geb. u. Gyn. Bd. 68. 81. Ätzstriktur der Cervix. Ztschr. f. Geb. u. Gyn. Bd. 68. - 82. Die Diagnose und Therapie der Genitaltuberkulose des Weibes. Dtsch. rr,ed. Woch. No. 23. - · 83. Über die Bedeutung der Phlegir,asia alba dolens. Ztschr. f. Geb. u. Gyn. Bd. 68. - 84. Pituitrin als Vorbereitung zum Kaiser-schnitt und als Mittel gegen Wehenschwäche. Zbl. f. Gyn. No. 39. - 85. Chorionepithelioma malignum nach Abort, mit Metastasen. Mon. f. Geb. u. Gyn. Bd. 34. - 86. Zur Hebosteotomie. Mon. f. Geb. u. Gyn. Bd. 34. -

Serie von Beckenausgangsmaßen. Mon. f. Geb. u. Gyn. Bd. 34. Infantilismus. Dtsch. med. Woch. No. 28. - 89 . Zur Eklampsie-frage. Mon. f. Geb. u. Gyn. - 90. Die Bedeutung der Anaerobier und die Behandlung der fieberhaften Abortes. Mon. f. Geb. u. Gyn. Bd. 34.

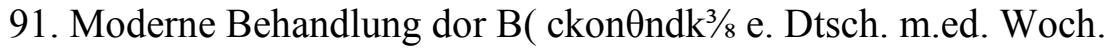

No. 35. - 92. Die Wanderniere als Teilerscheinung erworbener Enteroptose und ihre chirurgische Behandlung. Gyn. Rundsch. Bd. 5. 93. Kongenitale Nierenverlagerung. Dtsch. med. Woch. Bd. 37. - 94. Vorund Ausbildung der pretißischen Hebamm,en. 5. Tag. d. Verein. z. Ford. d. 
Hebammen'Wesens. - 95. Die Korpusamputation als typisches Sterilisationsverfahren bei Tuberkulose. Der Frauenarzt. No. 3. - 96. Tuboovarialtumor. Dtsch. med. Woch. No. 49. - 97. Klinische Wertung und Behandlung der Steißlagen. Mon. f. Geb. u. Gyn. Bd. 33. - 98. Über die' Bedeutung der Streptokokken und die Behandlung des fieberhaften Abortes. Ther. d. Gegenw. 11. - 1912: 99. Über Ovarialturnoren.- Dtsch. med. Woch. No. 3. - 100. Erweiterte K1/8rzmomoperation. Greifsw. Med. Verein. 20.1. 12. Dtsch. med. Woch. No. 12. - 101. RißverletZung der Scheide und Cervix mit anschließender "üterusruptur. Dtsch. med. Woch. No. 16.--102. Behandlung der Puerp $>$ ralinfektion mit Sireptococciisviridam. Dtsch. med. Woch. No. 66. -103. Kranrosis vulvae. Dtsch. med.Woch. No. 27. - 1(4. SchädelimpressionbeimNeugeborenen. Dtsch. rr,ed.Woch. No. 16.10 5. Tuber kmo seder inner en Genitalien und des Bauchf ells. Dtsch. med. Woch. No. 27. - - 106. Die Indikationen der chirurgischen Behandlung bei der Ge nitaltuberkulose. Mon. f. Geb. u. Gyn. Bd. 35. - 107. Zur Frage der prär. onitorischen Sympton.e der Thrombosen bzw. En.bolien. Dtsch. med. Paul Krömer $\uparrow$.

489

Wooh. No. 28. - 108. Über die Indikationen zu den endoVesikalen Opera -tionen und $i^{1} / 8 \mathrm{e}$ natürlichen Grenzen beim Weibe. Dtsch. med. Woch. No. 44. -109. Über die palliative Behandlung der Ovarialkarzinom $\beta$ und der anatomisch zweifelhaften Tumoren. Dtsch. rr.ed. Woch. No. 49. - 110. Überblick über die Behandlung der Gynatresien und über die Methoden zur plastischen Neubildung bei gänzlichem Mangel der Scheide. Dtsch. med. Woch. No. 50. Ill. Zur Frage des Frühaufstehens nach Operationen und Geburten. Med. Klin. - 112. Über pathogene Hefestämme. 6. Intern. Kongr. f. Geb. u. Gyn. Berlin. -· 1913; 113. Die plastische Neubildung der Scheide bei partielle $\prod 1$, und totalem Prolaps. Prakt. Ergebn. d. Geb. u. Gyn. 5. Jahrg. H. 1.-- 114. Einwirkung von Röntgen- und Mesothorium-strahlen auf maligne Neubildungen der Genitalien. Strahlenther. Bd. 3. H. 1. - - 115. Entstehung und Behandlung der Pyelitis beim Weibe. Dtsch. med. Woch. No. 10. - 116. Bedeutung des Röntgenapparates für die Gynä-kologieund Geburtshülfe. Dtsch. med. Woch. No. 14.-117. Mesothoriumeinwirkung auf genitaleNeubildungen. Verhandl. d· Dtsch. Ges. f. Gyn. 1913.- 118. Störungender Nierenfiinktion bei Eklampsie. Verhandl. d. Dtsch. Ges. f. Gyn. 1913. - 119. Zar Ätiologie bei Verschluß odor Unterbrechung des Uterus. Verh.d.Dtsch. Ges.f. Gyn.-- 120. Zur Ätiologie und Behandlung der Pyelitis gravidarum. Verhandl. d. Dtsch. Ges. f. Gyn. 1913. - 121. Eiter-niere bei Verschluß oder Unterbrechung des Ureters. Verhandl. d. Dtsch. Ges. f. Gyn. 1913. - 122. Tuberkulose der Vulva und Urethra. Verhandl. d. Dtsch. Ges. f. Gyn. 1913. - · 123. Beziehungen des Proc. vermiformis zu den weiblichen Genitalien. Dtsch. med. Woch. No. 50. - 124. Über seltene Geburtsstörungen. Korrespondenzbl. d. Ärztevereins d. Reg.-B日z. Stralsund. - 125. Mesothoriumeinwirkung auf Neubildungen der weiblichen Genitalien. Londoner Kongr. Zbl. f. Gyn. - 126. Beziehungen des \%oiro-poetischen Systems zu den weiblichen Genitalorganen mit Berücksichtigung der Baúchchirurgi $\theta$. Düsseldorf. Schmitz \& Olbertz. -— 191Ф: 127. Entstehung und Verlauf des Pseudomyxoma peritonei beim Weibe. Dtsch. med. Woch. No. 11. -

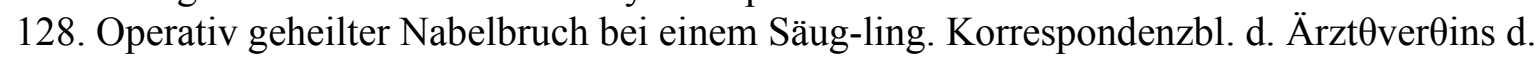
R日g.-Bez. Stralsund. -> 129. Heilung puerperaler Pyä $\pi$,ie d.urch Venenunterbind.ung. Bericht

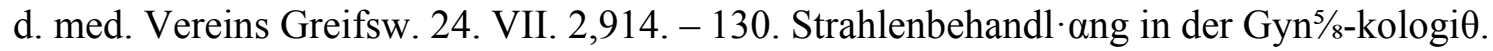
Korrespondenzbl. d. Ärztevereins d. Peg.-Bez. Stralsund. -I915: 
131. Senile Pyorrhoe und ihre B $\theta$ handlung. Dtsch. med. Woch. No. 8. 132. B Bhandlung von Karzinomrezidiven durch Röntgenbestrahlung. Munch, mod. Woch. - 1916: 133. Sarkomentwicklung in Myorr,en. Dtsch. med. Woch. No. 5. - $’$ 134. Einwirkung der Strahlentherapi $\theta$ auf Uteruskarzinom. Dtsch. med. Woch. No. 25. - 135 . Die medikamentöse Be handlung der Dysmenorrhoe. Ztschr. f. ärztl. Fortbild. No. 4. - 1917: 136. Der Einfluß der Strahlentberapie auf die Krebsb $\theta$ handlung und die Grenzen ihrer L $\theta$ istungsfähigkeit. Mon. f. Geb. u. Gyn. Bd. 46. - 137. Gefahren der Blasenmole. Dtsch. med. Woch. No. 9. - 138. GleichzeitigӨs Vorkommen von Neubildungen in Tube und Uterus. Dtsch. med. Woch. No. 11. - 139. Zur Bekämpfung der Geschlechtskrankheiten. Dtsch. med. Woch. No. 25. - 140. Sarkom der Scheide bei einem 4 Monato alten Säugling. Dtsch. med. Woch. No. 42. - 141. Die Behandlung krankhafter Genitalblutungen beim Weibe. Ztschr. f. ärztl. Fortbild. Ed. Schroeder, Greifs $\mathrm{Wa}^{1} / 8 \mathrm{~L}$ 